\title{
Two noncontiguous locations can be attended concurrently: Evidence from the attentional blink
}

\author{
JUN-ICHIRO KAWAHARA \\ Hiroshima University, Hiroshima, Japan \\ and \\ YUKI YAMADA \\ Kyushu University, Fukuoka, Japan
}

\begin{abstract}
When two targets (T1 and T2) are inserted in a rapid stream of visual distractors (RSVP), detection/ identification accuracy of T2 is impaired at intertarget lags shorter than about $500 \mathrm{msec}$. This phenomenon, the attentional blink (AB), has been regarded as a hallmark of the inability of the visual system to process multiple items. Yet, paradoxically, the AB is much reduced when T2 is presented directly after T1 (known as lag-1 sparing). Because lag-1 sparing is said to depend on observers' spatial attention being set to process the first target, we predicted that if observers are set to monitor two RSVP streams, they could process more than two items; that is, two instances of lag-1 sparing would be obtained concurrently. The results of three experiments indicated that this was the case. When observers searched for two targets in each of two synchronized RSVP streams, lag-1 sparing occurred concurrently in both streams. These results suggest that the visual system can handle up to four items at one moment under RSVP circumstances.
\end{abstract}

Human vision gives the impression of being extremely powerful because it occurs very quickly and effortlessly (see, e.g., Julesz, 1981; Thorpe, Fize, \& Marlot, 1996). It is also true, however, that visual performance is substantially restricted when multiple objects are processed in a brief moment. The phenomenon known as the attentional blink (AB; Shapiro, Arnell, \& Raymond, 1997) is a major example of such a deficit in visual processing (Marois \& Ivanoff, 2005). In a typical AB experiment, observers see two targets inserted into a rapid serial visual presentation (RSVP) stream of distractors. As expected, the magnitude of the AB deficit is most pronounced at shorter lags and is often maximized when the second target (T2) is presented directly after the first (T1), an ordinal position known as lag 1 (see, e.g., Joseph, Chun, \& Nakayama, 1997). It is generally agreed that this deficit represents the fact that the visual system can process only one item at a time. For example, Shapiro et al. claimed that this deficit occurs because attentional resources are depleted by the processing of $\mathrm{T} 1$, resulting in a scarcity of resources for $\mathrm{T} 2$ processing when the lag is short. The deficit is reduced at longer lags because the resources are released when the processing of T1 has been completed.

In the present study, we questioned whether the AB deficit really represents the processing limit of the visual sys-

J.-I.K. is now at the National Institute of Advanced Industrial Science and Technology. Correspondence relating to this article may be sent to J.-I. Kawahara, National Institute of Advanced Industrial Science and Technology, 1-1-1 Higashi, Tsukuba 305-8566, Japan (e-mail: jun.kawahara@, aist.go.jp). tem under RSVP circumstances, because it seems that the system can process more than one item in RSVP, in light of the following two sets of evidence. First, performance at lag 1 is sometimes relatively unimpaired, with the deficit occurring later. Potter, Chun, Banks, and Muckenhoupt (1998) termed such relatively unimpaired performance lag-1 sparing. To explain lag-1 sparing, researchers have elaborated the idea of sluggish closing of an attentional gating mechanism. The gate is said to open when T1 arrives and to close about $200 \mathrm{msec}$ later. When T2 appears immediately after $\mathrm{T} 1$, the two targets are processed together, and lag-1 sparing ensues. To explore the determinants of lag-1 sparing, Visser, Bischof, and Di Lollo (1999) conducted an extensive meta-analysis and found that lag-1 sparing occurs only when two targets differ in no more than one stimulus attribute. For example, when both targets are two alphabetical letters, lag-1 sparing occurs. In contrast, when two targets differ by more than one attribute (e.g., when T1 is a letter to be identified and T2 is a coherent motion patch for which the moving direction is to be reported), there is no lag-1 sparing; T2 accuracy is lowest at lag 1 and recovers as progressively as the lag increases.

This dependence of lag-1 sparing on the similarity of two targets can be explained by the idea of input filtering elaborated from the attentional-gating view (Visser, Bischof, \& Di Lollo, 1999). The input filter is a form of attentional set that gates visual inputs for further processing. In an AB sequence, the filter is initially set to optimize performance on T1. Thus, if two sequential targets belong to the same category, both will pass the filter and proceed for further processing, producing lag-1 sparing. If, however, they differ substantially, T2 will not pass the 
filter and will fail to be identified (i.e., no lag-1 sparing). Therefore, it is possible to process more than one item if the same attentional set can be used for separate targets.

Second, it has been shown using various types of task that the capacity of visual short-term memory is about four items (see, e.g., Luck \& Vogel, 1997), which is double the estimate in the $\mathrm{AB}$ literature. Thus, making use of the idea of input filtering, we surmise that if the input filter is set optimally for identifying targets, the visual system can handle more than one or two items at a time. To illustrate, consider two synchronized RSVP streams, each containing two targets. The T1s in both streams appear concurrently. Both $\mathrm{T} 2 \mathrm{~s}$ also appear concurrently, with a variable lag after T1. If the processing limit is one or two, as has been assumed in the $\mathrm{AB}$ paradigm, $\mathrm{T} 2$ performance would be poor across all lags, and it would not recover at longer lags when both T1s had been correctly identified, because the T1s would have exhausted the processing capacity. Conversely, if the processing limit is larger, T2 performance would recover at longer lags, even when both T1s had been identified. More importantly, lag-1 sparing would be obtained simultaneously in both streams. Given that in several studies observers have been able to attend two noncontiguous locations at a time (see, e.g., Awh \& Pashler, 2000; Kramer \& Hahn, 1995), it is possible that filters could be set concurrently at different locations. Müller, Malinowski, Gruber, and Hillyard (2003) also demonstrated electrophysiologically that sustained attention can be split concurrently between RSVP streams in noncontiguous locations. These findings suggest that observers can establish an attentional set such that they can monitor two locations simultaneously. If so, two instances of lag-1 sparing, occurring concurrently at different locations, should be obtained. Such findings would seriously put into question the prevalent belief that the capacity limit in the $\mathrm{AB}$ paradigm is essentially one. In fact, the limit may be contingent on the attentional set observers adopt.

\section{EXPERIMENT 1}

\section{Method}

Observers. Twelve experimentally naive undergraduates at Hiroshima University participated for extra course credit. All reported normal or corrected-to-normal visual acuity.

Stimuli and Apparatus. The target letters were chosen randomly from the English alphabet, excluding the characters $I, O, Q$, and $Z$. The first letters presented in the two streams (the T1s) consisted of the same character in half of the trials and of different characters in the remaining half. The second two targets always consisted of different characters, neither of which were the same as the first two targets. The distractor sequence consisted of digits. The letters and digits subtended approximately $0.76^{\circ}$ of visual angle in height and width. The stimulus display consisted of a central fixation point and two synchronized RSVP streams of characters, one to the left and the other to the right of the fixation point. The center-to-center distance between the two streams was $3.4^{\circ}$ of visual angle. The stimuli were displayed in black on a white background on a computer monitor.

Procedure. The observers initiated each trial by pressing the space bar. After a delay of $500 \mathrm{msec}$, two synchronized RSVP streams were displayed, each of which contained a variable number of digits (distractors) and two letters (T1 and T2). Each item in the stream was displayed for $100 \mathrm{msec}$ and was followed immediately by the next item, with no interstimulus interval. In any given trial, the distractors in each stream were selected randomly from the set of digits (0-9), with the constraint that the selected digit differed from the one immediately preceding it. Also, the distractors in the two streams in any given frame were never the same. The number of distractors preceding the first target varied from 5 to 10 , determined randomly on each trial. T1s in the left and right streams appeared simultaneously. T2s were also presented simultaneously in the two streams, at one of five lags $(100,200,300,500$, or $700 \mathrm{msec})$. The observers made three responses: In the first, an observer indicated whether the T1s were the same or different by pressing one of two assigned keys. We adopted this same-different judgment to reduce the possibility that performance could be impaired by forgetting in the period leading to the response (the pattern of results did not change when, in a pilot study, a T1 identification task was used instead). The second and the third responses consisted of identifying the two $\mathrm{T} 2 \mathrm{~s}$ by pressing the corresponding keys on the keyboard. The RSVP stream of distractors continued to be displayed throughout the lag period. After the T2s, two more distractor frames appeared in each stream. There were 20 practice trials before the experimental session, which consisted of 300 trials.

\section{Results and Discussion}

In this and all subsequent experiments, estimates of $\mathrm{T} 2$ identification were based only on those trials in which the $\mathrm{T} 1$ task was performed correctly. The segmented lines in Figure 1 show the mean accuracy of identification of the second target across lags, separately for the left and right streams. An ANOVA was performed with two withinsubjects factors: stream (left or right) and lag (100, 200, $300,500$, or $700 \mathrm{msec})$. The analysis revealed a significant effect of $\operatorname{lag}\left[F(4,44)=31.55, M S_{\mathrm{e}}=3,105.21, p<.001\right]$, but neither the effect of stream $\left[F(1,11)=1.40, M S_{\mathrm{e}}=\right.$

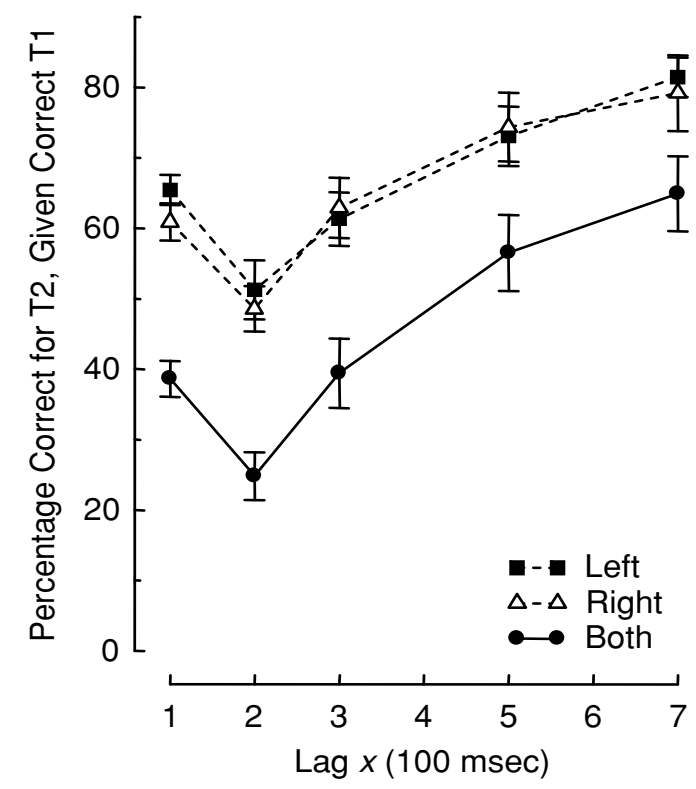

Figure 1. Results of Experiment 1: Mean percentages of correct reporting of the second targets, given accurate reporting of the first targets. The squares and triangles indicate second-target performance in the left and right streams, respectively. The filled disks indicate second-target performance when the responses were correct in both streams. The error bars indicate standard errors for each condition. 
$181.79, p>.2]$ nor the interaction effect $[F(4,44)<1]$ was significant. Post hoc comparisons of the main effect of lag indicated that $\mathrm{T} 2$ performance at lag 1 was significantly higher than at lag $2[t(44)=4.10, p<.001]$. Because the effect of stream was not significant in this or any other statistical analysis in the present work, we combined the scores for the left and the right streams in all further analyses. Correct report of T1, averaged across all lags, was $85.5 \%$. A two-way ANOVA (lag $\times$ location) revealed a significant effect of lag $\left[F(4,44)=32.6, M S_{\mathrm{e}}=660.9\right.$, $p<.001]$. There was no effect of location or interaction between the two factors.

The results clearly show that lag-1 sparing occurred in both streams. When interpreting this finding, however, we need to consider the possibility that the presence of lag-1 sparing in both streams might be a consequence of averaging across trials. Namely, it is possible that, in any given trial, lag-1 sparing occurred in only one stream, and never in both streams, but that this was obscured by averaging the results across trials. To check this possibility, we analyzed only those trials in which both T2s were identified correctly (solid line in Figure 1). An ANOVA once again revealed a significant effect of lag $\left[F(4,44)=28.94, M S_{\mathrm{e}}=\right.$ $3,003.6, p<.001]$, and post hoc comparisons indicated that $\mathrm{T} 2$ performance at lag 1 was significantly higher than at lag $2[t(44)=3.25, p<.005]$. This confirms that lag-1 sparing occurred concurrently in both streams.

This pattern of results reveals a new aspect of capacity limitation in the $\mathrm{AB}$, in that the number of items that can be processed at one time is not just one but up to four; that is, it is not innately fixed, but varies depending on the attentional set. When observers were set the task of monitoring four letter targets in independent streams, lag-1 sparing occurred for each stream. In this sense, the visual system can handle two T1s concurrently at a given moment. Moreover, if two T2s appear immediately after the T1s, the T2s can also be processed. One might argue, however, that this finding does not mean that the observers perceived four targets; they may have perceived two sets of spatially grouped targets (i.e., two T1s and two T2s). In terms of a metaphorical "spotlight" of attention, (see, e.g., Posner, 1980), observers might apply a larger area of spotlight to encompass both streams, so that the two $\mathrm{T} 1 \mathrm{~s}$ are included in a single attentional event. If this is true, then items presented in the region between the two streams would also be expected to pass the filter. Experiment 2 examined this possibility.

\section{EXPERIMENT 2}

We presented two targets (letters) in each of two RSVP streams of distractors (digits). Each T2 could appear in one of three locations (Figure 2). First, in the same condition, two targets were presented within each stream. The second was termed the inward condition; here, the T2s were displaced horizontally toward fixation. Finally, the third was labeled the outward condition, in which T2s were displaced horizontally away from fixation.

We predicted that if observers applied a spotlight of attention to a larger area, encompassing both streams (so the two T1s were taken into a single attentional event), then lag-1 sparing would occur in both the same and inward conditions. In contrast, if the observers prepared an input filter to spatially monitor two individual RSVP streams, then the region between the two streams would be unattended, and lag-1 sparing would be found in the same condition only. These predictions were based on evidence that change in the targets' physical location does not eliminate lag-1 sparing, as long as the targets are in the attentional beam (Shih, 2000). The outward condition was included to explore the outer boundary of the spatial extent of input filtering, should concurrent monitoring of noncontiguous locations be established.

\section{Method}

The stimuli, apparatus, and procedures were the same as in Experiment 1 , except for the following. T2s were presented in one of three locations: within the two streams (same condition) or displaced toward

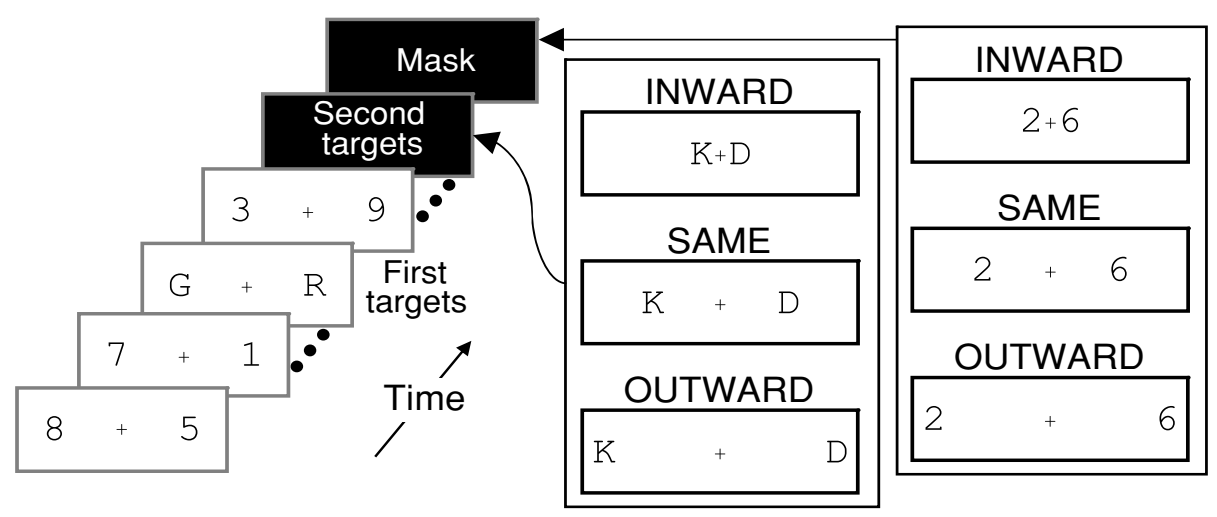

Figure 2. Schematic representation of stimuli in Experiment 2. Each of the first targets was masked, in all instances, by a trailing distractor presented in the stream. Each of the second targets was masked by two trailing distractors that were presented at the same locations as the second targets. Note that in the inward and outward conditions, the two T2s and the two distractors to mask the T1s were presented in the same frame when the lag was 1. 
(inward condition) or away from (outward condition) fixation. The intertarget distances in the inward, same, and outward conditions were $1.2^{\circ}, 3.4^{\circ}$, and $5.6^{\circ}$, respectively. In the inward and outward conditions, each $\mathrm{T} 1$ was masked by a distractor, and only the T2s and their trailing distractors were away from the streams; all items before the T2s were presented in the same locations as the T1s. In the inward and outward conditions, both the two T2s and the two distractors to mask the $\mathrm{T} 1 \mathrm{~s}$ were presented in the same frame when the lag was 1 . The three conditions (same, inward, and outward) and the five lags (100, 200, 300,500 , and $700 \mathrm{msec}$ ) were combined factorially and presented for a total of 300 trials. Thirteen experimentally naive undergraduates who reported normal or corrected-to-normal visual acuity participated, and they were given 20 practice trials prior to the experimental session.

\section{Results}

Figure 3 shows the percentages of correct identifications of both second targets concurrently as a function of lag, averaged across all observers and presented separately for each second-target location. A two-way ANOVA with the within-subjects factors lag $(100,200,300,500$, or $700 \mathrm{msec}$ ) and location (same, inward, or outward) revealed a significant effect of lag $\left[F(4,48)=48.76, M S_{\mathrm{e}}=\right.$ $12,308.9, p<.001]$ and a significant interaction between lag and location $\left[F(8,96)=5.22, M S_{\mathrm{e}}=1,113.6, p<\right.$ $.001]$. Multiple post hoc comparisons using Ryan's (1960) method indicated that lag-1 sparing was obtained only in the same condition; performance at lag 1 was significantly higher than at lag 2 in the same condition $[t(144)=2.40$, $p<.05]$, but performance at lag 1 was lower than at lag 2 in both the inward $[t(144)=3.38, p<.001]$ and outward $[t(144)=2.57, p<.05]$ conditions.

Correct identification of the first target, averaged across all lags, was $84.0 \%$. A two-way ANOVA (lag $\times$

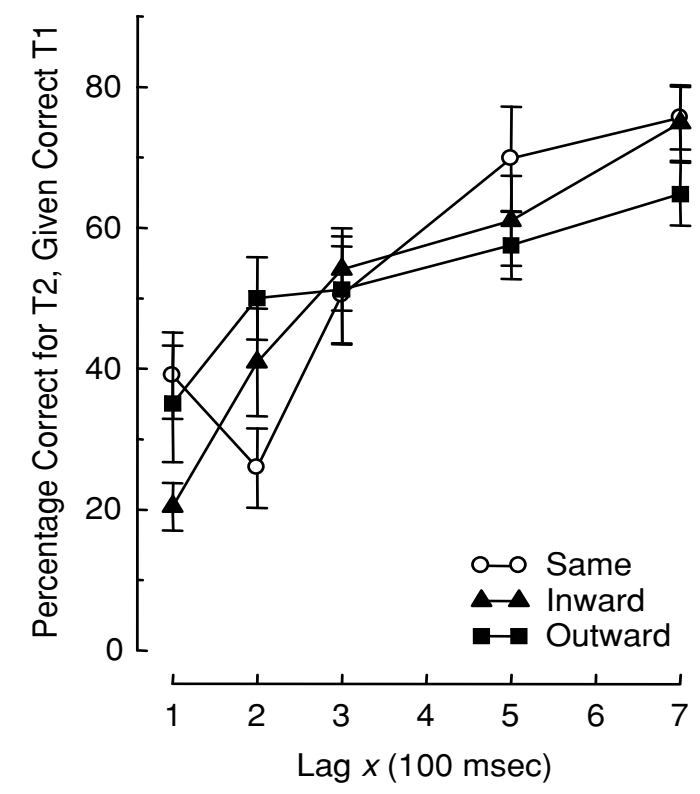

Figure 3. Results of Experiment 2: Mean percentages of correct reporting of the second targets when the first-target responses were correct in both streams. The error bars indicate standard errors for each condition. location) revealed a significant effect of lag $[F(4,48)=$ $\left.6.05, M S_{\mathrm{e}}=260.8, p<.001\right]$. There was no effect of location or interaction between the two factors.

\section{Discussion}

Our finding that lag-1 sparing occurred in the same condition but not the inward condition suggests that the observers were able to monitor two individual RSVP streams at noncontiguous locations. If two T1s were spatially grouped, as in a large attentional spotlight encompassing both RSVP streams, lag-1 sparing should also have been obtained in the inward condition. Lag-1 sparing was not evidenced in the outward condition, which suggests that attentional set was finely tuned to the locations at which the T1s were presented. We conclude that the visual system can maintain attentional sets at different, noncontiguous locations and that the intervening locations can remain unattended.

It should be noted that performance in the outward condition at the shorter lags was more accurate than in the inward condition. Although counterintuitive, this pattern of results is consistent with those of letter reading studies (see, e.g., Mackworth, 1965). As a potential cause for such an asymmetric effect, Bouma (1973) proposed that visual interference of a masking type, characteristic of eccentric vision and predominantly acting toward the fovea, plays a role. It was also true that the inward targets were hemmed in on two sides, but the outward targets only suffered crowding on one side. However, these effects of eccentric advantage and crowding were not prevalent and did not occur at longer lags.

\section{EXPERIMENT 2A}

Experiment 2A was conducted to dismiss a concern that the absence of lag-1 sparing in the inward and outward conditions in Experiment 1 might be due to the presence of four items (two T2s and two T1 masks) at lag 1 only in these conditions. This experiment replicated Experiment 2, but in the same condition the T2s were accompanied by four additional distractors (i.e., two betweenstream and two outside-stream distractors) when the lag was 1 . If the presence of additional distractors in Experiment 2 was critical for the absence of lag-1 sparing, lag-1 sparing should not be obtained in any condition. All other aspects of the stimuli and procedure were the same as in Experiment 2. Sixteen experimentally naive undergraduates participated.

\section{Results and Discussion}

The results are presented in Figure 4. A two-way ANOVA with two within-subjects factors (lag and location) revealed a significant effect of $\operatorname{lag}[F(4,56)=72.03$, $\left.M S_{\mathrm{e}}=17,429.8, p<.001\right]$ and a significant interaction between lag and location $\left[F(8,112)=4.97, M S_{\mathrm{e}}=463.4\right.$, $p<.001]$. Post hoc comparisons indicated that lag-1 sparing was obtained only in the same condition; performance at lag 1 was significantly higher than at lag 2 in the same 


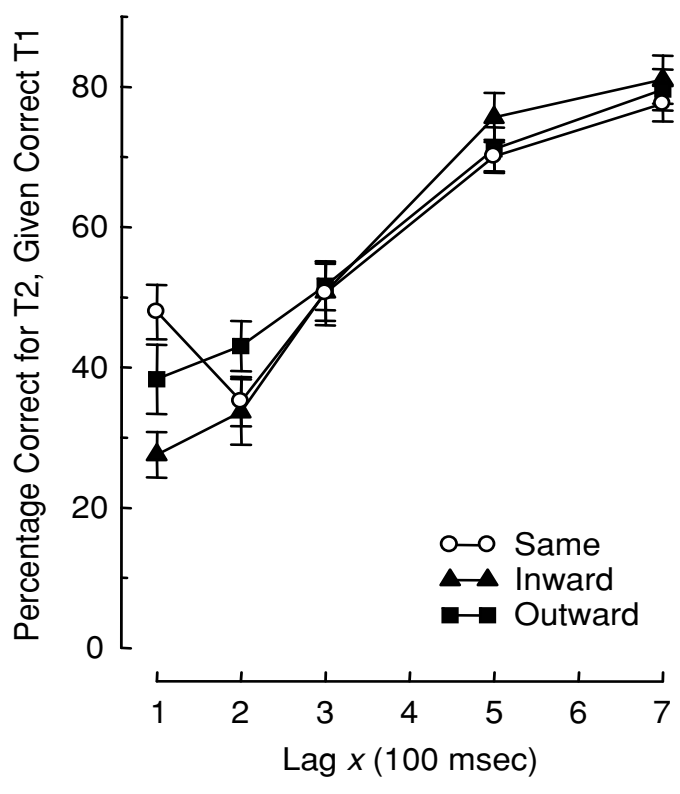

Figure 4. Results of Experiment 2A: Mean percentages of correct reporting of the second targets when the first-target responses were correct in both streams. The error bars indicate standard errors for each condition.

condition $[t(168)=2.93, p<.01]$. The results clearly indicate that the presence of additional distractors at lag 1 did not lead to the absence of lag-1 sparing in Experiment 2.

\section{GENERAL DISCUSSION}

The purpose of the present study was to examine whether the capacity limit for identification in RSVP streams is constrained to one or at most two items. Experiment 1 showed that lag- 1 sparing could occur concurrently in two streams, suggesting that it is possible to identify up to four targets concurrently in the AB situation. This shows that the capacity limit measured in the $A B$ paradigm is not essentially one, but is contingent on the attentional setting when viewing the display. In other words, existing $\mathrm{AB}$ studies have shown that observers can barely attend to more than one target at a time; the present study has shown that this is because they were set to monitor only one RSVP stream. When they are set to monitor two streams, as in the present study, it is possible to process up to four items during the same period of time.

The finding that observers can process up to four items concurrently at lag 1 when they are monitoring two streams is consistent with the idea that lag-1 sparing is independent of the AB (Visser, Bischof, \& Di Lollo, 1999). In their meta-analysis, Visser, Bischof, and Di Lollo found that the magnitudes of lag-1 sparing and of the $\mathrm{AB}$ were not correlated, and they suggested that these two phenomena reflect the involvement of two different but sequential stages: conceptually driven control at the input level (i.e., an input filter) and stimulus-driven control at higher processing levels (i.e., an attention-demanding stage). On the basis of this view, we surmise that the second target entered the subsequent processing stage for each stream at lag 1, since the setting of the input filter was to monitor streams in the present study. This resulted in an apparent doubling of capacity in comparison with the $\mathrm{AB}$ as traditionally conceived.

The present finding is also consistent with the idea of contingent involuntary orienting (Folk \& Remington, 1998), which hypothesizes that the processing of incoming stimuli hinges on top-down attentional settings. Previous studies have shown that stimuli that share the same feature (e.g., color) as the current attentional setting are processed automatically. The present study showed that when observers monitor two streams, targets sharing the same spatial location as the current attentional setting can be processed concurrently for the two streams.

Another intriguing finding is that in Experiment 2, lag-1 sparing occurred only in the same condition. In conjunction with recent findings that lag-1 sparing can be regarded as an index of the maintenance of the spatial focus of attention in the $\mathrm{AB}$, we argue that the present study provides converging evidence for split foci of attention (Awh \& Pashler, 2000; Kramer \& Hahn, 1995), although earlier studies reported that lag-1 sparing occurs only when two targets are presented at the same location (Visser, Zuvic, Bischof, \& Di Lollo, 1999). Specifically, Jefferies, Kawahara, Ghorashi, and Di Lollo (2005) and Shih (2000) found that lag-1 sparing occurs even when two targets appear at different locations if observers are attentionally focused on the correct T1 location, and these researchers have shown that how observers prepare their spatial attention for the first target is critical in this respect.

Moreover, the present results imply a potential dissociation between the nature of attention effects observed during the $\mathrm{AB}$ period (i.e., lag-1 sparing) and outside of the $\mathrm{AB}$ (i.e., performance at lag 7); there is a possibility that attentional foci can be split at lag 1, but not immediately afterward. ${ }^{1}$ For example, two distinct attentional mechanisms, as suggested by Vogel, Luck, and Shapiro (1998) - one that is specific to spatial selection and another that is more general - could be involved in the manifestation of the "split foci" results in Experiment 2. It is, however, premature to conclude that the present results indicate attentional split only at lag 1 , because it is necessary to demonstrate that the present procedure is sensitive to spatial attention effects during later lags. Including a probe detection task within the present task would be a way to test this sensitivity. Another way to check the spatial sensitivity would be to use the shooting line illusion, which is known as a sensitive measure of spatial attention during the AB (Kawahara, 2002). In this illusion, if spatial attention is engaged, a static line presented near T2 at lag 7 is seen as if it were drawn from the side close to $T 2$. In this sense, the present study also presented the possibility that the AB/RSVP paradigm offers a powerful tool for investigating the nature of two different mechanisms of attention involved in the identification of temporally and spatially distributed targets. 


\section{REFERENCES}

Awh, E., \& PASHLER, H. (2000). Evidence for split attentional foci. Journal of Experimental Psychology: Human Perception \& Performance, 26, 834-846.

Bouma, H. (1973). Visual interference in the parafoveal recognition of initial and final letters of words. Vision Research, 13, 767-782.

Folk, C. L., \& Remington, R. (1998). Selectivity in distraction by irrelevant featural singletons: Evidence for two forms of attentional capture. Journal of Experimental Psychology: Human Perception \& Performance, 24, 847-858.

JefFeries, L. N., Kawahara, J.-I., Ghorashi, S. M. S., \& Di Lollo, V. (2005). Dynamic spatial tuning of attentional focus in the attentional blink. Manuscript submitted for publication.

Joseph, J. S., Chun, M. M., \& Nakayama, K. (1997). Attentional requirements in a "preattentive" feature search task. Nature, 387, 805807.

Julesz, B. (1981). Textons, the elements of texture perception, and their interactions. Nature, 290, 91-97.

KaWAHARA, J.-I. (2002). Facilitation of local information processing in the attentional blink as indexed by the shooting line illusion. Psychological Research, 66, 116-123.

Kramer, A. F., \& HAHN, S. (1995). Splitting the beam: Distribution of attention over noncontiguous regions of the visual field. Psychological Science, 6, 381-386.

Luck, S. J., \& Vogel, E. K. (1997). The capacity of visual working memory for features and conjunctions. Nature, 390, 279-281.

Mackworth, N. H. (1965). Visual noise causes tunnel vision. Psychonomic Science, 3, 67-68.

MaroIs, R., \& IVANOFF, J. (2005). Capacity limits of information processing in the brain. Trends in Cognitive Sciences, 9, 296-305.

Müller, M. M., Malinowski, P., Gruber, T., \& Hillyard, S. A. (2003). Sustained division of the attentional spotlight. Nature, 424, 309-312.
Posner, M. I. (1980). Orienting of attention. Quarterly Journal of Experimental Psychology, 43, 307-318.

Potter, M. C., Chun, M. M., Banks, B. S., \& Muckenhoupt, M. (1998). Two attentional deficits in serial target search: The visual attentional blink and an amodal task-switch deficit. Journal of Experimental Psychology: Learning, Memory, \& Cognition, 24, 979-992.

RYAN, T. (1960). Significance test for multiple comparison of proportions, variances, and other statistics. Psychological Bulletin, 57, 318328.

Shapiro, K. L., Arnell, K. M., \& Raymond, J. E. (1997). The attentional blink. Trends in Cognitive Sciences, 1, 291-296.

SHIH, S. (2000). Recall of two visual targets embedded in RSVP streams of distractors depends on their temporal and spatial relationship. Perception \& Psychophysics, 62, 1348-1355.

Thorpe, S., Fize, D., \& Marlot, C. (1996). Speed of processing in the human visual system. Nature, 381, 520-522.

Visser, T. A. W., Bischof, W. F., \& Di Lollo, V. (1999). Attentional switching in spatial and nonspatial domains: Evidence from the attentional blink. Psychological Bulletin, 125, 458-469.

Visser, T. A. [W.], Zuvic, S. M., BischoF, W. F., \& Di Lollo, V. (1999). The attentional blink with targets in different spatial locations. Psychonomic Bulletin \& Review, 6, 432-436.

Vogel, E. K., LuCK, S. J., \& SHAPIRo, K. L. (1998). Electrophysiological evidence for a postperceptual locus of suppression during the attentional blink. Journal of Experimental Psychology: Human Perception \& Performance, 24, 1656-1674.

\section{NOTE}

1. We thank Dr. Ed Awh for pointing out this possibility.

(Manuscript received July 13, 2004; revision accepted for publication January 11, 2006.) 